gung das Netzwerk verfügen muss. Damit verbunden ist die Suche nach der optimalen Delegation von Entscheidungen. Eine Schlüsselrolle könnte den jeweiligen Bundestagsmandaten insbesondere deren ressortübergreifender Erarbeitung - zufallen, indem diese mit konkreten Vorgaben für die vernetzte Zusammenarbeit versehen werden. Eine weitere Schlüsselrolle hat das gemeinsame Lagebild, denn der Bedarf an Instrumenten oder Strukturen, die eine aggregierte Lagebeurteilung und Früherkennung und damit gemeinsame Bewertungen und Zielformulierungen ermöglichen, ist unbestritten.

Für einige Zeit schien das Thema Vernetzte Sicherheit in Vergessenheit geraten zu sein. Nun wird es wieder thematisiert: ob vom ehemaligen Bundespräsidenten Christian Wulff mit seinen Einlassungen bei seinem letzten Besuch in Afghanistan; ${ }^{15}$

15 Vgl. Bundespräsident Christian Wulff, http://www.reservistenverband.de/ php/evewa2.php?d=1319572169\&menu=0105\&newsid=9841\&g2=1 (Zugriff 12.11.2011) von Nikolaus Schneider, dem Vorsitzenden des Rates der EKD in seinem Vortrag Auf dem Weg zu einem gerechten Frieden? in Bonn; ${ }^{16}$ oder von Verteidigungsminister de Maizière auf der Handelsblattkonferenz ${ }^{17}$ - sie alle haben sich in jüngster Zeit mit den Herausforderungen Vernetzter Sicherheit auseinandergesetzt und damit der Debatte um dieses wichtige Thema neue Impulse gegeben. Die lebendige Diskussion um gemeinsame Strategien und Ziele sowie deren praktische Umsetzung im Krisenmanagement bietet den erfolgversprechendsten Ansatz, den vernetzten Ansatz voranzutreiben.
16 Vgl. Schneider, Nikolaus, a.a.O.

17 BMVg, Rede von BundesverteidigungsministerThomas de Maizière anlässlich der 8. Handelsblattkonferenz, a.a.O.

\title{
Sicherheitspolitik zwischen immanenten Tücken und Gestaltungsspielräumen - einige kategoriale Reflexionen
}

\author{
Sabine Jaberg*
}

\begin{abstract}
Networked security participates in the logic of security, a logic that includes a number of traps: selfishness, boundlessness, dramatization, and escalation. But security policy is a social practice that can be influenced by various actors. The traps can be partially deactivated by undertaking self-reflective turns, drawing borders, and restricting the security label to extreme personal violence. Since the end of the Cold War, German policy has gone in the opposite direction. Even constitutional barriers have been torn down. For peace research, security is both a risky and an indispensable term. There are two ways out of this dilemma: strengthening an alternative peace discourse (bypass strategy) or developing a concept of security that is more geared towards peace (détente strategy).
\end{abstract}

Keywords: Logic of security, Comprehensive Approach, German foreign and security policy Sicherheitslogik, vernetzte Sicherheit, deutsche Außen- und Sicherheitspolitik

\section{Vernetzte Sicherheit - ein zweifelhafter Hoffnungsträger}

$\mathrm{D}$ as Weißbuch der Bundesregierung von 2006 etabliert das Schlagwort von der vernetzten Sicherheit. ${ }^{1}$ Mit dieser Innovation verspricht sich die hohe Politik konzeptionelle Antworten auf jene Problemlagen, die sie seit Beginn der 1990er Jahre im Lichte eines weiten Sicherheitsverständnisses zeichnet. ${ }^{2}$ Jeder Herausforderung

* Dr. Sabine Jaberg, Dozentin für Politikwissenschaft am FB Human- und Sozialwissenschaften an der Führungsakademie der Bundeswehr in Hamburg; Privatdozentin für Friedensforschung an der Westfälischen Wilhelms-Universität Münster.

1 Vgl.: Weißbuch 2006 zur Sicherheitspolitik Deutschlands und zur Zukunft der Bundeswehr. Berlin: Bundesministerium der Verteidigung, 2006 (zit.: Weißbuch 2006).

2 Bereits in den Verteidigungspolitischen Richtlinien von 1992 heißt es: „Sicherheitspolitik [läßt sich] weder inhaltlich noch geographisch eingrenzen." Verteidigungspolitische Richtlinien. Bonn: Bundesminister der Verteidigung, 1992, S. 16. sei mit einem maßgeschneiderten Mix ziviler und militärischer Ansätze zu begegnen - so die Grundidee. Teile der wissenschaftlichen Zunft loben schon länger die Überwindung des „Begriffsdualismus von Sicherheit und Frieden“. ${ }^{3}$ Ihres Erachtens subsumiere der Sicherheitsbegriff des politischen Establishments mittlerweile ,all die Werte [...], die einst für den ,positiven Friedensbegriff“ reklamiert wurden“4. Für Christopher Daase und Philipp Offermann macht es demnach weder theoretisch noch praktisch einen Unterschied, ob eine Angelegenheit im sicherheits- oder friedenspolitischen Paradigma beleuchtet wird. Demgegenüber vertritt dieser Beitrag die These, dass Sicherheitspolitik ungeachtet ihrer konkreten Beschaffenheit bestimmten paradigmatischen Eigenheiten

3 Daase, Christopher/Offermann, Philipp: Subkulturen der Sicherheit. Die Münchner Sicherheitskonferenz und die Münchner Friedenskonferenz im Vergleich, in: Sicherheit und Frieden (S+F), 2/2011, S. 84-89; hier: S. 85. 4 Ebd., S. 85. 
unterliegt. Daher sollen zuerst die Tücken der Sicherheitslogik herausgestellt werden, um danach Gestaltungsspielräume der Politik aufzuzeigen und zu überprüfen, ob und inwieweit Deutschland sie nutzt..

\section{Tücken der Sicherheitslogik}

Auch die Auseinandersetzung mit einem spezifischen Ansatz vernetzter Sicherheit verlangt eine kategoriale Reflexion auf den allgemeinen Sicherheitsbegriff. Dies gilt zumindest vor dem Hintergrund der Annahme, dass Sicherheitskonzepte bei aller Erscheinungsvielfalt gleichsam unentrinnbare Gemeinsamkeiten aufweisen, die der theoretischen Prägung durch die realistische Denkschule entspringen. Deren philosophischer Kronzeuge Thomas Hobbes erhebt nicht nur das nach Selbsterhaltung strebende zweckrationale Individuum zum letzten Geltungsgrund staatlicher Macht, sondern er setzt damit auch das Sicherheitsdenken frei. Dessen Logik lässt sich aus seiner Schrift „Leviathan“5 (1651) rekonstruieren - einschließlich der immanenten Tücken: Selbstbezüglichkeit, Grenzenlosigkeit sowie Dramatisierung und Eskalation.

\subsection{Selbstbezüglichkeit}

Zu den Eigenheiten des Sicherheitsparadigmas gehört die unausweichliche Selbstbezüglichkeit. So rechtfertigt sich nach Hobbes der Staat allein aus seiner Schutzfunktion „gegen auswärtige und innere Feinde“. ${ }^{6}$ Als Schutzpatron seiner Bevölkerung erhebt er sich zum entscheidenden Ausgangs- und Referenzpunkt. Es geht um seine (nationale) Sicherheit, aber auch um seine (nationale) Macht, seine (nationalen) Interessen.

Das angeführte Zitat zeigt des Weiteren: Sicherheitsdenken fokussiert auf Akteure - und zwar auf eine Weise, die den jeweils anderen zuerst unter Feindverdacht nimmt. Damit generiert sie zwei blinde Flecken: Zum einen gerät der eigene Beitrag zur Entstehung dessen, was als Bedrohung wahrgenommen wird, aus dem Sichtfeld. Zum anderen gilt der Feind nicht nur als Symptom, sondern er erscheint auch als Urheber der Gefahr. Dieser personalisierte Blick vermag tieferliegende strukturelle Ursachen kaum zu erfassen.

\subsection{Grenzenlosigkeit}

Das Sicherheitsparadigma entwickelt aus sich selbst heraus keine Grenzen. Dies betrifft erstens die Wahl der Mittel, denen sich der Staat bedienen darf, „denn wem die Erhaltung [...] der allgemeinen Sicherheit obliegt, dem muß“ - so Hobbes - „auch der freie Gebrauch aller dazu dienlichen Mittel zugestanden werden“7. Dazu zählt er nicht zuletzt die Entscheidung über Krieg und Frieden. So müsse „die höchste Gewalt Krieg gegen

5 Hobbes, Thomas: Leviathan. Erster und zweiter Teil [1651]. Übersetzung von Jacob Peter Mayer. Nachwort von Malte Diesselhorst. Stuttgart: Philip Reclam jun, 1980.

6 Ebd., S. 155

7 Ebd., S. 163. andere Staaten nach Gutdünken beschließen oder Frieden mit ihnen machen, das heißt beurteilen können, ob ein Krieg ihrem Staate vorteilhaft oder nachteilig sein wird oder nicht“8. Die einzigen beschränkenden Instanzen wären demnach funktionale Angemessenheit und das einzelfallbezogene KostenNutzen-Kalkül.

Zweitens fehlen der Sicherheitslogik zeitliche Limits. Vielmehr kommt nach Hobbes dem Staat das „Recht“ zu, „sowohl in der Gefahr selbst wie zu ihrer Abwendung schon vorher das Nötige zu veranstalten, damit die Bürger im Innern und von außen her in Sicherheit leben “9 (Herv. SJ). Folglich findet Sicherheitspolitik nicht nur reaktiv gegen akute Gefahren statt, sondern sie richtet sich bereits ,präventiv‘ gegen rein potenzielle Bedrohungen.

Drittens beschränkt Hobbes weder Sektoren noch geografische Reichweite der Sicherheitspolitik. Grundsätzlich kann sich ihre Logik in jedes Themenfeld und zu jedem Ort vorschieben. Doch wie lässt sich der spezifische Sicherheitsgehalt einer Angelegenheit dann identifizieren? Hobbes verzichtet auf intersubjektiv überprüfbare Kriterien. Stattdessen legt er die Entscheidung darüber, „was zur Erhaltung oder zur Störung des Friedens“ im Sinne des Schutzes der Bürger vor innerer und äußerer Gefahr „dienen kann“ ins subjektive Ermessen der „höchsten Gewalt“10. Beispielhaft erwähnt er die „Beurteilung aller Meinungen und Lehren, weil diese nicht selten Grund und Ursprung von Uneinigkeit und Bürgerkrieg sind“11. Hier zeigt sich: Die Allgegenwärtigkeit der Sicherheitspolitik geht mit ihrer radikalen Subjektivierung einher. Das Streben nach absoluter Kontrolle leistet totalitären Tendenzen zumindest Vorschub.

\subsection{Dramatisierung und Eskalation}

Sicherheitspolitik neigt paradigmatisch bedingt zur Dramatisierung, schließlich zielt sie auf das Überleben des Staats als Schutzpatron seiner Bevölkerung. Das bedeutet im Umkehrschluss: Das Prädikat ,sicherheitsrelevant' kommuniziert zumindest implizit eine existentielle Dimension. Bei extensiver Verwendung erscheint das bezeichnete Problem unter Umständen gravierender, als es tatsächlich ist. Mit dieser Dramatisierung der Lage geht eine Eskalation im Handeln einher: Denn im Notwehrmodus gilt der Rückgriff auf gewaltsame Instrumente zumindest als zulässig, wenn nicht gar als geboten. Diesen Mechanismus nennt die ,Kopenhagener Schule‘ in ihrem Securitization-Ansatz „security form“12 oder auch „grammar of security “13.

8 Ebd., S. 162.

9 Ebd., S. $160 \mathrm{f}$.

10 Ebd., S. 161.

11 Ebd., S. 161.

12 Buzan, Barry/Waever, Ole/Wilde, Jaap de: Security. A New Framework for Analysis. Boulder; London: Lynne Rienner, 1998, S. 33.

13 Ebd., S. 33. 


\section{Gestaltungsspielräume der Sicherheitspolitik}

Sicherheitspolitik unterliegt wie jede soziale Praxis nicht unausweichlichen Naturgesetzen, sondern kennt Gestaltungsmöglichkeiten. Somit können ihre paradigmenbedingten Tücken gezielt angegangen werden: Gegen Selbstbezüglichkeit hilft Blickfeldveränderung. Grenzenlosigkeit verlangt nach Errichtung von Schranken. Und eine sparsame Verwendung des Sicherheitsetiketts trägt zur Entschärfung des Dramatisierungsund Eskalationspotenzials bei. Diese Gegenstrategien gilt es im Folgenden näher zu entfalten. Damit steht auch die Frage im Raum: Inwieweit macht Deutschlands amtliche Politik von diesen Optionen Gebrauch? Zur Beantwortung stellt das nach wie vor gültige Weißbuch 2006 das erste Bezugsdokument dar.

\subsection{Blickfeldveränderungen}

Der Selbstbezüglichkeit wirken zwei Strategien zur Veränderung des Blickfelds entgegen: Zum einen ließe sie sich innerhalb des realistischen Theorierahmens durch Reflexion auf ein wohlverstandenes Eigeninteresse abfedern. Zum anderen könnte ein Theoriensprung versucht werden, der den Sicherheitsbegriff aus dem Realismus herauslöst und in einen eher idealistischen Kontext einfügt. Dies käme einer Neuerfindung gleich.

\subsubsection{Reflexive Aufklärung des Eigeninteresses}

Die Selbstbezüglichkeit des Sicherheitsdenkens lässt sich innerhalb einer realistischen Weltsicht zwar nicht überwinden, aber doch mildern. Hierzu bietet sich insbesondere die Kategorie internationaler Sicherheit an - sei es im Weltmaßstab als, globale Sicherheit' oder im geografisch begrenzten Raum als ,regionale Sicherheit'. Anders als beim nationalen Pendant geht es hier nicht um die isolierte Betrachtung eines einzelnen Staats, sondern dieser wird Teil einer Gesamtkonstellation. Eine solche Einbindung nötigt ihn systematisch, um der eigenen Sicherheit willen auch Interessen und Perspektiven anderer Akteure zu berücksichtigen. Diesen kommt aus Sicht des jeweiligen Staats aber keine eigenständige, sondern lediglich eine von seinem Kalkül abhängige Wertigkeit zu. Dies zeigt sich auch im Weißbuch: Es spricht im Kontext sowohl der Weiterverbreitung von Massenvernichtungswaffen und ihrer Trägermittel als auch der Energieproblematik beispielhaft von einer Bedrohung globaler Sicherheit, wobei in beiden Fällen die Auswirkungen auf Deutschland besondere Betonung finden. ${ }^{14}$ Jedoch handelt es sich eher um beiläufig erwähnte Stichwörter als um entfaltete Konzepte.

Je aufgeklärter Sicherheitspolitik wird, desto eher erscheint es möglich, sich ihrer immanenten Logik zu entziehen: Blinde Flecken wie der eigene Beitrag zur Problementstehung lassen sich zumindest dann ausleuchten, wenn man um sie weiß. Und die verkürzte Perspektive auf den Akteur kann auf zugrundeliegende Strukturen verlängert werden. Voraussetzung ist jedoch eine bewusste reflexive Wende der Sicherheitspolitik. Diese unterbleibt jedoch im Weißbuch. Das dort unterstellte, Wir ‘ also Deutschland und die gesamte ,westliche Welt" - kommt

14 Vgl.: Weißbuch 2006, S. 25 und S. 27. vor allem als Opfer von Krisen und Bedrohungen vor, die durch irgendwen anderswo verursacht sind (z.B. transnationaler Terrorismus, Migration). Allenfalls gerieren ,wir' uns mit leicht idealistischem Einschlag noch als Helfer, die ,anderen“ bei der Bewältigung ihrer Krisen etwa durch Förderung von Menschenrechten und Entwicklungschancen zur Seite springen. Als Mitverursacher von Krisen geraten ,wir' hingegen nicht ins Visier, wie das folgende Beispiel illustriert: Bei den im Weißbuch beklagten „Störungen der Rohstoff- und Warenströme [...] durch zunehmende Piraterie"15 endet der sicherheitspolitische Blick beim räuberischen Akt bzw. bei seinen unmittelbaren Urhebern: den Piraten. Der eigene Anteil am Geschehen, zu dem etwa die Zerstörung der Subsistenzfischerei vor Afrikas Küsten durch hochtechnisierte Fangflotten auch aus Europa zählt, gerät hingegen aus dem Sichtfeld. ${ }^{16}$

\subsubsection{Neuerfindung des Sicherheitsbegriffs}

Während sich das moderate Plädoyer für eine aufgeklärte Sicherheitspolitik noch innerhalb des angestammten Theorierahmens bewegte, bestünde eine radikalere Alternative darin, den Sicherheitsbegriff aus dem Realismus herauszulösen und in einen anderen Theoriekontext einzufügen. Diese Überlegung liegt letztlich dem Human-security-Ansatz zugrunde. All seine Varianten verfügen über zwei Gemeinsamkeiten: Sie fokussieren ohne Umweg über den Staat direkt auf den einzelnen Menschen. Und sie ersetzen das Prinzip der Exklusivität durch das der Inklusivität. Mithin geht es nicht mehr um die besondere Sicherheit von Angehörigen eines bestimmten Staatsverbands, sondern um die Sicherheit eines jeden Menschen. Offenbar unternimmt das Konzept der human security eine sicherheitspolitische Reformulierung altbekannter friedenswissenschaftlicher Basiskategorien: ${ }^{17}$ In Johan Galtungs Begriffsrepertoire ausgedrückt entspräche die enge ,kanadische' Variante freedom from fear dem ,negativen Frieden“ im Sinne einer Abwesenheit personaler Großgewalt, während die weite ,japanische‘ Variante freedom from want ähnlich dem ,positiven Frieden' den Aspekt nachhaltiger menschlicher Entwicklung betont und zusätzliche lebensrelevante Faktoren einbezieht. Hierzu zählen Ökonomie, Gesundheit, Ökologie, Gesellschaft und Politik. Demgegenüber fokussiert die dritte ,europäische“ Variante wieder etwas enger auf Menschenrechte und Rechtssicherheit. ${ }^{18}$

Zwar ist es etwa im Konzept menschlicher Sicherheit durchaus möglich, die Grenze zwischen idealistischem Friedensdenken und realistischem Sicherheitsdenken zu überwinden. Allerdings dürfte ein altruistisch anmutender Sicherheitsbegriff, der sich aus Perspektive des wohlhabenden und befriedeten ,Westens zunächst auf andere zu beziehen scheint, eher als Ergänzung denn als Alternative zum herkömmlichen - eigenbezüglichen Sicherheitsbegriff denkbar sein. Denn nach Franz-Xaver Kauf-

15 Ebd., S. 26

16 Vgl.: Mahnkopf, Birgit: Piratenhatz am Horn von Afrika. Zur politischen Ökonomie eines Piratenkonflikts und seiner geopolitischen Bedeutung, in Internationale Politik und Gesellschaft, 1/2010, S. 58-81; hier: S. 61-64.

17 Vgl.: Galtung, Johan: Strukturelle Gewalt. Beiträge zur Friedens- und Konfliktforschung. Reinbek: Rowohlt, 1975, S. 7-59.

18 Vgl.: Ulbert, Cornelia/Werthes, Sascha: Menschliche Sicherheit - Der Stein der Weisen für globale und regionale Verantwortung?, in: dies. (Hrsg.) Menschliche Sicherheit. Globale Herausforderungen und regionale Perspektiven. Baden-Baden: Nomos-Verlagsgesellschaft, 2008. (Eine Welt. Texte der Stiftung Entwicklung und Frieden; 21.) S. 13-27; hier: S. 17-20. 
mann handelt es sich beim Streben nach (individueller) Sicherheit zumindest in entwickelten Gesellschaften um ein quasi konstitutives menschliches Attribut. ${ }^{19}$ Wie dem auch sei: ,Menschliche Sicherheit' taucht im Weißbuch nicht auf - der ,Aktionsplan Zivile Krisenprävention“ der Bundesregierung erwähnt sie immerhin einmal, wenn auch eher beiläufig. ${ }^{20}$

\subsection{Errichtung von Schranken}

Immanentes Begrenzungspotenzial kennt eine ungehegte Sicherheitspolitik kaum: Es erschöpft sich in negativen Kosten-Nutzen-Kalkülen bzw. im Verdacht auf unangemessene Zweck-Mittel-Relationen. Bereits im Konstrukt internationaler Sicherheit sind es aus Sicht des Akteurs letztlich die Interessen und Fähigkeiten der anderen, die dem eigenen Sicherheitsstreben Grenzen setzen. Dauerhafte Schranken lassen sich demnach nicht den Eigenbewegungen der Sicherheitspolitik entnehmen, sondern sie müssen ihr von außen gesetzt werden. Diese können sich - eingedenk der bereits angestellten kategorialen Reflexionen ${ }^{21}$ - auf die prinzipiell für zulässig erachteten Mittel, den anvisierten Zeitrahmen, die betroffenen Sektoren und die geografische Reichweite beziehen.

Als begrenzende Instanz kommt vornehmlich das Recht in Betracht. Mit dem Grundgesetz verfügt Deutschland über eine Verfassung, die einer wildwüchsigen, friedensunverträglichen Sicherheitspolitik Einhalt gebietet. ${ }^{22} \mathrm{Ob}$ aus rechtlichen Normen auch praktische Schranken werden, hängt nicht zuletzt von der Bereitschaft der Politik ab, sich solchen Bändigungsstrategien dauerhaft zu unterwerfen. Wieweit ist dies gegenwärtig noch der Fall?

\subsubsection{Konditionierung des Militäreinsatzes auf Verteidigungslagen}

Das Grundgesetz erlaubt in Artikel 87a Absatz 1 die Aufstellung von Streitkräften weiterhin nur zu einem Zweck: Verteidigung. Streng genommen deckt es einzig den Einsatz der Streitkräfte gegen einen stattfindenden oder unmittelbar bevorstehenden bewaffneten Angriff auf bundesdeutsches Territorium ausdrücklich ab. Bereits ein militärischer Beistand für angegriffene Bündnispartner lässt sich nur durch interpretatorische Einbeziehung des Entstehungskontextes der Wehrverfassung (1956) rechtfertigen. Denn zeitgleich zur Aufstellung der Streitkräfte wird die Bundesrepublik Mitglied in der Nordatlantischen Vertragsorganisation (NATO) und der Westeuropäischen Union (WEU). Zumindest Letztere verlangt den militärischen Beistand, den der Gesetzgeber offenbar zu leisten willens war.

19 Vgl.: Kaufmann, Franz-Xaver: Sicherheit als soziologisches und sozialpolitisches Problem. Untersuchungen zu einer Wertidee hochdifferenzierter Gesellschaften. 24 Tabellen, 9 Übersichten. 2., umgearbeitete Aufl. Stuttgart: Ferdinand Enke Verlag, 1973, S. 10-14 und S. 29.

20 Vgl.: Aktionsplan „Zivile Krisenprävention, Konfliktlösung und Friedenskonsolidierung“. Berlin: Bundesregierung, 2004, S. 57.

21 Vgl.: Abschnitt 2. dieses Beitrags.

22 Vgl.: Jaberg, Sabine: Wehe, wehe, wehe, wenn ich auf das Ende sehe ... Zur Begründung eines friedenswissenschaftlichen Standpunkts zum Norm-EmpirieProblem bei Auslandseinsätzen der Bundeswehr, in: dies./Biehl, Heiko/Mohrmann, Günter/Tomforde, Maren (Hrsg.): Auslandseinsätze der Bundeswehr. Sozialwissenschaftliche Analysen, Diagnosen und Perspektiven. Berlin: Duncker \& Humblot, 2009. (Sozialwissenschaftliche Schriften 47) S. 295-325.
Zwar räumt Artikel 24 Absatz 2 dem Bund von Beginn an die Möglichkeit des Beitritts zu - nicht näher definierten - Systemen gegenseitiger kollektiver Sicherheit ein, allerdings finden Streitkräfte hier keine Erwähnung. Damit wird die Vorgabe des im Zuge der Notstandsgesetze 1968 eingefügten Artikels 87a Absatz 2 nicht eingelöst, wonach der Streitkräfteeinsatz jenseits der Verteidigung nur bei ausdrücklicher Erwähnung erlaubt sei. Mithin beschränkt der Wortlaut der Verfassung Sicherheitspolitik unterhalb der Schwelle zur Selbstverteidigung auf nichtmilitärische Mittel.

Die Praxis seit Ende des globalen Macht- und Systemkonflikts (1989/90) hebt diese Konditionierung faktisch auf. Fortan betreibt Deutschland eine aktive Einsatzpolitik: von der Umsetzung der durch die Vereinten Nationen beschlossenen Sanktionen gegen Jugoslawien (1992) über die Beteiligung am eigenmächtig beschlossenen Kosovokrieg der NATO (1999) bis hin zum Engagement in Afghanistan in unterschiedlichen Missionen (seit 2002). Dabei kann sich die Politik seit 1994 auf das Streitkräfteurteil des Bundesverfassungsgerichts stützen, das militärisches Engagement im Rahmen kollektiver Sicherheitssysteme grundsätzlich als erlaubt ansieht, zu denen es auch klassische Militärbündnisse wie die NATO rechnet.

Der Ansatz vernetzter Sicherheit fängt diese Dekonditionierung des Militärischen nunmehr konzeptionell auf. Gemäß Weißbuch ergebe sich im Einsatz zum einen „die Notwendigkeit einer vernetzten Zusammenarbeit mit militärischen wie zivilen Akteuren im nationalen und internationalen Rahmen "23 (Herv. SJ). Zum anderen gehe es um „einen umfassenden vernetzten Ansatz, der zivile und militärische Instrumente wirksam verbin-

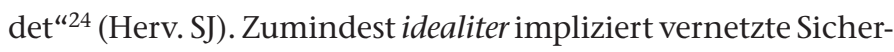
heit die Schaffung eines zivil-militärischen Gesamtakteurs und eines zivil-militärischen Instrumentenpools, aus dem sich Politik im Einzelfall die als zweckdienlich erachteten Komponenten zusammenstellt: Dort, wo zivile Kräfte bereits wirken, stellt sich die Frage nach dem möglichen militärischen Beitrag. Und dort, wo Streitkräfte bereits agieren, sucht vernetzte Sicherheit zivile Unterstützung. Ihr ist das militärische Instrument zumindest als Option stets eingeschrieben. Diese Omnipräsenz befördert die Mutation der Bundeswehr vom Spezialinstrument der Verteidigung zum ,normalen' Mittel allgemeiner Außenpolitik. Dazu fehlt ihr jedoch der verfassungsrechtliche Auftrag.

\subsubsection{Befristung des Selbstverteidigungsrechts}

Sicherheitspolitik kennt paradigmenbedingt keine zeitlichen Limits. Das Grundgesetz setzt ihr aber zumindest beim Rückgriff auf die Streitkräfte Schranken: Zwar geht es über ein eng umrissenes Selbstverteidigungsrecht hinaus, das auf die Abwehr einer gegenwärtigen militärischen Attacke gegen bundesdeutsches Territorium beschränkt wäre. Vielmehr bezieht es auch einen unmittelbar bevorstehenden Angriff in den Verteidigungsfall ein. Außerhalb einer derart akuten Gefahrensituation darf die Bundeswehr nach Verfassungslage auch dann nicht eingesetzt werden, wenn dies wie in der Nationalen Sicherheitsstrategie

23 Weißbuch 2006, S. 98.

24 Ebd., S. 15. 
der USA von 2002 als antizipatorische Selbstverteidigung gegen eine sich künftig entwickelnde Bedrohung gedeutet würde. ${ }^{25}$

Trotz zurückhaltender Wortwahl erweist sich die deutsche Sicherheitsdoktrin der letzten Jahre an die amerikanische Auffassung aber zusehends anschlussfähig. So deckt das Weißbuch ein präventives Verständnis von Sicherheitspolitik mit ab: „Sicherheitsvorsorge kann [...] am wirksamsten durch Frühwarnung und präventives Handeln gewährleistet werden“26 (Herv. SJ). Mithin greift sie bereits in einem Stadium, in welchem ,Risiken und Bedrohungen“ die Sicherheit Deutschlands noch nicht akut beeinträchtigen. Die „Bundeswehr mit ihrem gesamten Fähigkeitsspektrum“ gilt längst als Mittel zur „frühzeitigen Konflikterkennung, Prävention und Konfliktlösung “27. Und die Beistandsverpflichtung für Bündnispartner wird ganz im Sinne ,antizipatorischer Nothilfe‘ auf Krisen und Konflikte ausgedehnt, „die zu einer konkreten Bedrohung eskalieren können “28 (Herv. SJ). Und genau dieses hatten die USA im Vorfeld ihres völkerrechtswidrigen Kriegs gegen den Irak 2003 immer wieder behauptet. Hier hat die Politik verfassungsrechtliche Fesseln abgestreift.

\subsubsection{Sektorale und geografische Einhegungen}

Sicherheitspolitik vermag sich grundsätzlich in jedes Themenfeld und an jeden Ort vorzuschieben. Hier setzt das Grundgesetz keine ausdrückliche Grenze. Zwar erwähnt es Sicherheit ohne nähere Definition nur in zwei Kontexten: der öffentlichen Sicherheit (Artikel 13 und 35) und dem kollektiven Sicherheitssystem (Artikel 24). Es findet sich aber kein Verfassungsvorbehalt wie beim Streitkräfteeinsatz, der einer ausdrücklichen Erwähnung im Grundgesetz bedarf. Sektorale wie geografische Schranken müssen daher anderweitig gesetzt werden - sei es durch Diskurse zugunsten eines engen Sicherheitsbegriffs, sei es durch Selbstanpassungen der Politik an die perzipierten Rahmenbedingungen.

Während des globalen Macht- und Systemkonflikts dominierte in der Bundesrepublik Deutschland in Wissenschaft wie politischer Praxis ein enges Verständnis von Sicherheit. Es lief vereinfacht gesprochen darauf hinaus, die Gefahr eines atomaren Schlagabtauschs zwischen den gegnerischen Blöcken, der nicht zuletzt auf eigenem Territorium geführt worden wäre, durch Gewährleistung der Abschreckungsstabilität zu bannen oder wenigstens zu minimieren. Allerdings darf auch nicht übersehen werden, dass der enge Sicherheitsbegriff und seine militärischen Auswüchse große Teile des politischen wie gesellschaftlichen Alltags überformten. Hierfür hat Dieter Senghaas den - durchaus umstrittenen - Begriff „organisierter Friedlosigkeit" ${ }^{29}$ geprägt.

Mit Ende der Blockkonfrontation entfällt für die Sicherheitspolitik des vereinigten Deutschlands der begrenzende Fokus.

25 Vgl.: Die nationale Sicherheitsstrategie der Vereinigten Staaten von Amerika. Washington: Weißes Haus, 2002 (Übersetzung vom Amerika-Dienst), Kapitel V, S. 23

26 Weißbuch 2006, S. 29.

27 Ebd., S. 20

28 Ebd., S. 75.

29 Senghaas, Dieter: Abschreckung und Frieden. Studien zur Kritik organisierter Friedlosigkeit. Frankfurt/M.: Fischer Taschenbuch Verlag, 1972 (Bücher des Wissens 6157).
Flankiert durch entsprechende wissenschaftliche Plädoyers ${ }^{30}$ setzt sich in öffentlichen Strategiedokumenten seit Beginn der 1990er Jahre ein sektoral wie geografisch entgrenztes Sicherheitsverständnis durch, das sich auch im Weißbuch 2006 niederschlägt. Die fehlende Konturierung dokumentiert sich bereits im Verzicht auf eine klare Sicherheitsdefinition zugunsten einer diffusen Beschreibung von Sicherheitspolitik. Deren Ziel bestehe letztlich ganz allgemein darin, „die Interessen unseres Landes zu wahren“31. Entsprechend greift Sicherheitspolitik über das Ziel, „die Souveränität und die Unversehrtheit des deutschen Staatsgebietes zu sichern “32 unter anderem auf die Wahrung des Wohlstands aus. Die problemfeldspezifischen Auffächerungen erweitern diese Bandbreite eher, als dass sie sie beschneiden: ${ }^{33}$ Vorbeugung und Bewältigung regionaler Krisen und Konflikte, Begegnung globaler Herausforderungen wie dem internationalen Terrorismus und der Weiterverbreitung von Massenvernichtungswaffen, Achtung der Menschenrechte und Stärkung der internationalen Ordnung auf Grundlage des Völkerrechts, Förderung des freien und ungehinderten Welthandels sowie Hilfe zur Überwindung der Kluft zwischen armen und reichen Weltregionen. Darüber hinaus attestiert das Weißbuch an unterschiedlichen Stellen weiteren Themenfeldern sicherheitspolitische Relevanz. Hierzu gehören beispielsweise illegaler Waffen- und Drogenhandel, fragile Staatlichkeit, Transport- und Kommunikationswege, Ressourcen, Energiesicherheit, Migration, Pandemien und Seuchen, Schutz natürlicher Lebensgrundlagen, friedenserhaltende und friedensschaffende Einsätze der Vereinten Nationen, Überwindung des Nord-Süd-Gefälles und nachhaltige Entwicklung. ${ }^{34}$ Nahezu jeder Themenbereich steht unter Sicherheitsverdacht.

Daher kann auch die geografische Entgrenzung kaum verwundern. Laut Weißbuch besitzen Risiken und Bedrohungen für die deutsche Sicherheit „ihren Ursprung in regionalen und globalen Entwicklungen oftmals weit jenseits des europäischen Stabilitätsraums“35 (Herv. SJ). In Kombination mit dem Anspruch auf präventive Sicherheitspolitik plädiert das Weißbuch folgerichtig dafür, diesen Herausforderungen „rechtzeitig dort zu begegnen, wo sie entstehen"36 (Herv. SJ). In der Konsequenz bedeutet dies einen Militäreinsatz ,oftmals‘ weit außerhalb Europas.

Ein derart ausuferndes Sicherheitsverständnis neigt aber, wie in Hobbes' Plädoyer für ein Entscheidungsmonopol erkennbar, ${ }^{37}$ zu Subjektivität und totalitären Tendenzen. Beidem lässt sich dadurch entgegenwirken, dass außer der Exekutiven auch Legislative und Judikative Mitwirkungsrechte erhalten. Das Grundgesetz liefert hierzu Ansätze. So räumt es der Legislative beim Streitkräfteeinsatz eine prominente Stellung ein: Die Entscheidung über den Verteidigungsfall trifft auf Antrag der Bundesregierung der Bundestag mit Zustimmung des Bundesrats. Bei Verhinderung geht die Feststellungkompetenz gerade nicht auf die Exekutive, sondern auf den Gemeinsamen Ausschuss als

30 Vgl.: Daase, Christopher: Der erweiterte Sicherheitsbegriff. Frankfurt/M.: Projekt Sicherheitskultur im Wandel an der Goethe-Universität Frankfurt, 2010 (Working Paper 1/2010).

31 Weißbuch 2006, S. 28.

32 Ebd., S. 28.

33 Vgl.: Ebd., S. 28.

34 Vgl.: Ebd., S. 23-27 und S. 57.

35 Ebd., S. 29.

36 Ebd., S. 23.

37 Vgl.: Abschnitt 2.2 dieses Beitrags. 
Abbildung 1: Anatomie des Sicherheitsbegriffs im Weißbuch 2006

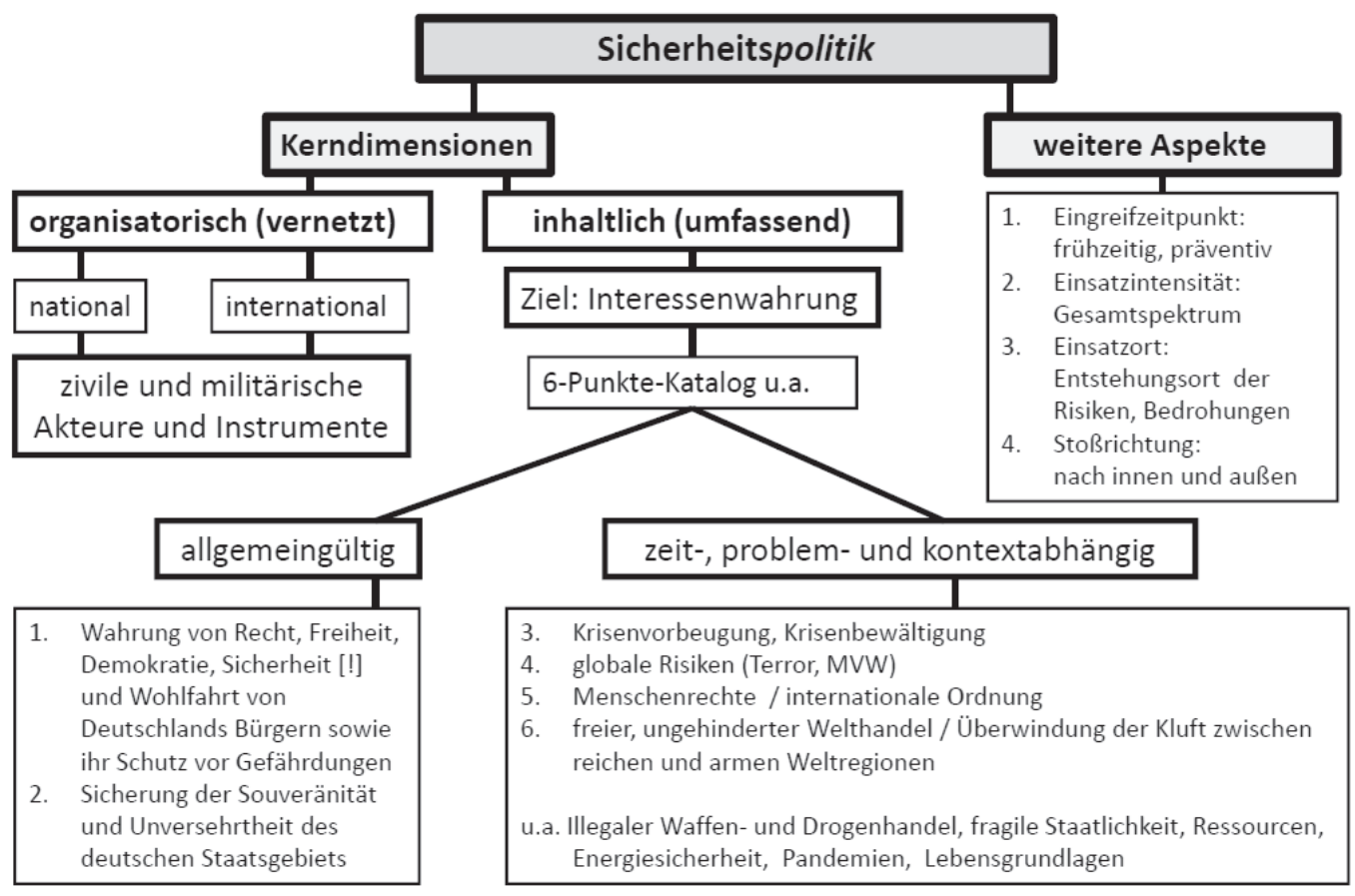

Schrumpfparlament über. Darüber hinaus verpflichtet Artikel 115a die Politik auf eine tendenziell objektivierbare materielle Legaldefinition des Verteidigungsfalls als stattfindender oder unmittelbar bevorstehender Angriff auf Bundesgebiet.

Nach einer verfassungsrechtlich äußerst fragwürdigen Entsendepraxis der schwarz-gelben Regierungskoalition Anfang der 1990er Jahre bejaht das Urteil des Bundesverfassungsgerichts 1994 zwar die Möglichkeit, Streitkräfte in friedenswahrenden Kollektivsystemen auch außerhalb des Verteidigungsfalls einzusetzen. Es verlangt aber die konstitutive Zustimmung des Bundestags. Das Parlamentsbeteiligungsgesetz von 2005 strickt die Mitwirkungsrechte der Legislativen sogar relativ engmaschig. ${ }^{38}$ Allerdings versucht die Exekutive punktuell, Lücken oder Ungenauigkeiten zu nutzen bzw. aufzutun - wie anlässlich des Libyenkriegs geschehen, als die Bundeswehr im Februar 2011 Ausländer evakuierte und einige Soldaten in den involvierten NATO-Stäben verblieben, ohne dass die Legislative in irgendeiner Form damit befasst worden wäre. ${ }^{39}$ Wenngleich das Weißbuch das Parlamentsbeteiligungsgesetz unangetastet lässt, bleibt ungewiss, ob das letzte Wort in dieser Angelegenheit gesprochen ist. Gemäß den neuen Verteidigungspolitischen Richtlinien von 2011 sei zu analysieren, ob und inwieweit die Zusammenarbeit in Bündnissen „rechtlichen Anpassungsbedarf "40 nach sich ziehe. Vorschläge zur Beschneidung parlamentarischer Mitwirkungsrechte könnten folgen.

38 Vgl.: Jaberg, Sabine: Sag mir wo ...? Auf der Suche nach der grundgesetzlichen Friedensnorm beim Streitkräfteeinsatz. Hamburg: Institut für Friedensforschung und Sicherheitspolitik an der Universität Hamburg, 2006 (Hamburger Beiträge zur Friedensforschung und Sicherheitspolitik 143), S. 58-73.

39 Vgl.: Löwenstein, Stephan: Bewaffnete Unternehmungen, in: Frankfurter Allgemeine Zeitung vom 23. August 2011.

40 Bundesministerium der Verteidigung: Verteidigungspolitische Richtlinien. Berlin, 27. Mai 2011, S. 6.

\subsection{Refokussierung des Sicherheitsdiskurses}

Sicherheitspolitik zielt paradigmenimmanent auf das Überleben des jeweiligen Akteurs. Die damit einhergehende Gefahr einer Dramatisierung der Lage und einer Eskalation des Handelns in den Notwehrmodus lässt sich zwar nicht bannen, aber reduzieren. Das verlangt jedoch eine Refokussierung des Sicherheitsdiskurses auf existentielle Bedrohungen in Form personaler Großgewalt. Macht, Einfluss und Wohlstand stellen andersartige Kategorien dar. Bei ihnen geht es nicht um Existenz, sondern um Gestaltung und Komfort. Sie wären allein durch kluge Politik, nicht durch militärische Gewalt zu verwirklichen. Damit fände die Auseinandersetzung mit den meisten Problemen im politischen ,Normalmodus‘ statt, der nichtmilitärische Lösungen favorisiert.

Im Weißbuch geschieht das Gegenteil: Zum einen scheint es so, als sollten möglichst viele Angelegenheiten in unterschiedlichen Politikfeldern aktuellen oder potenziellen Sicherheitsstatus erhalten (securitization).$^{41}$ Dies erleichtert der Politik den Rückgriff auf militärische Mittel dann, wenn sie ihn aufgrund strategischer Kalküle oder situativer Erwägungen für nützlich erachtet. ${ }^{42}$ Zum anderen finden sich Dramatisierungen einzelner Politikfelder. Beispielsweise sieht das Weißbuch die Integrationsfähigkeit europäischer Gesellschaften durch „Ströme“" ${ }^{43}$ von Flüchtlingen überfordert. Oder das begonnene Bild zu Ende gemalt: In der bevorstehenden Überflutung drohen Bürgerinnen und Bürger der Einwanderungsgesellschaften unterzugehen. Der nahegelegte Ausweg besteht präventiv darin, solche Ströme noch vor den eigenen Grenzen aufzuhalten bzw. umzuleiten - gegebenenfalls unter Einsatz personaler

41 Vgl.: Abschnitt 3.2.3 dieses Beitrags.

42 Vgl.: Abschnitt 2.3 dieses Beitrags.

43 Weißbuch 2006, S. 27. 
Gewalt. Reaktiv setzt er auf rigorose Abschiebung. Die StromMetapher im Weißbuch geht aber an der Realität vorbei: Nicht die Industrie-, sondern die Entwicklungsländer sind von den Flüchtlingsbewegungen mit etwa achtzig Prozent am meisten betroffen. Sie stemmen - gemessen an der Leistungsfähigkeit der Ökonomien - eine überproportionale Last. ${ }^{44}$

\section{Fazit}

Der friedenswissenschaftliche Anspruch gerät in ein Spannungsfeld: Auf der einen Seite sind der Sicherheitslogik einige Tücken eingeschrieben, die den Verzicht auf den Sicherheitsbegriff nahelegen. Auf der anderen Seite gilt das Streben nach Sicherheit durchaus als konstitutives menschliches Attribut, was sie zur quasi unvermeidbaren Kategorie erhebt. Im Umgang mit dem Spannungsfeld bestehen zwei miteinander kombinierbare Optionen: Bei einer Umgehungsstrategie ginge es darum, die im Sicherheitsparadigma konstruierten Problemfelder wie z.B. Migrationsbewegungen im Lichte eines gerechten Friedens neu zu formulieren. Damit würde eine konkurrierende Sichtweise zumindest ins Spiel gebracht. Als Entspannungsstrategie hingegen böte sich Karlheinz Koppes Ansatz einer „friedensverträgliche[n] Sicherheitspolitik“45 an. Mit Blick auf die identifizierten Tücken bedeutete dies dreierlei:

Erstens müsste der Selbstbezüglichkeit gegengesteuert werden. Dazu böte sich ein alternativer Sicherheitsbegriff wie human security an. Da es sich hier aber eher um eine Ergänzung als um eine Verdrängung traditioneller Ansätze handeln dürfte, spränge dieser Versuch zu kurz. Daher bedürfte es vor allem einer reflexiven Wende in der herkömmlichen Sicherheitspolitik. Diese äußerte sich zum einen im Ringen um Empathie für Bedürfnisse anderer Akteure sowie in der Bewusstmachung des eigenen Anteils an der Konfliktgenese. Zum anderen müsste der verkürzte Blick auf den Akteur als sichtbarer Gefahrenträger hin zu tieferliegenden Strukturen verlängert werden. Letztere bestehen nicht selten in einer asymmetrischen Machtverteilung, die

44 Vgl.: Global Trends 2010. Genf: United Nations High Commissioner for Refugees, 2011.

45 Koppe, Karlheinz: Exkurs zum Friedensbegriff in der Friedenswissenschaft, in: Senghaas, Dieter/ders. (Hrsg.): Friedensforschung in Deutschland. Lagebeurteilung und Perspektiven für die neunziger Jahre. Dokumentation eines Kolloquiums Berlin 17.-19. Juli 1990. Bonn: Arbeitsstelle Friedensforschung Bonn, 1990, S. 106-110; hier: S. 110. zur global unterschiedlichen Verteilung von Lebens- und Gestaltungschancen führt. Erinnert sei hier nur an die Abschottung des europäischen Markts gegenüber konkurrenzfähigen Produkten aus Afrika. ${ }^{46}$

Zweitens gälte es, der seit Ende des globalen Macht- und Systemkonflikts entfesselten Sicherheitslogik erneut Schranken zu setzen. Dazu müsste den einschlägigen Vorgaben des Grundgesetzes faktisch wieder zur Geltung verholfen werden. Die Verfassung erlaubt die Aufstellung der Streitkräfte zu einem einzigen Zweck: der Verteidigung, wobei sie den Verteidigungsfall auf einen stattfindenden oder unmittelbar bevorstehenden bewaffneten Angriff auf Bundesgebiet sowohl inhaltlich begrenzt als auch zeitlich befristet. Allenfalls der Beistand für angegriffene Bündnispartner lässt sich noch rechtfertigen. Militärische Generalprävention ist auch unter dem Etikett antizipatorischer Selbstverteidigung bzw. antizipatorischer Nothilfe verboten. Sie verstößt gegen die Norm des absoluten Gewaltverbots und damit auch gegen das Grundgesetz, das sich zum Völkerrecht bekennt. Sicherheitspolitische Praxis und Doktrinen sind folglich in den Bereich des Zulässigen zurückzuführen. Dazu gehört auch der Abschied vom vernetzten Sicherheitsansatz, der die militärische Option ins ,normale‘ Repertoire der Politik einspeist. Allerdings kann es Situationen geben, in denen ein Einsatz bewaffneter Kräfte dem Schutz ,fremder‘ Bevölkerungen etwa vor Völkermord dienen könnte. Dies wirft jedoch die Frage nach dem angemessenen Rahmen auf. Da es sich letztlich um Aufgaben einer Weltinnenpolitik handelt, sollte die ihr angemessene Organisation - nämlich die Vereinten Nationen - mit den erforderlichen Bordmitteln ausgestattet werden.

Drittens ginge es um Entschärfung des Dramatisierungs- und Eskalationspotenzials. Dies geschähe zum einen durch Refokussierung des Sicherheitsdiskurses auf existentielle Gefährdungen in Form personaler Großgewalt. Zum anderen wären Metaphern zu meiden, die wie die ,Flüchtlingsströme‘ Bedrohungen überzeichnen und unterschwellig Ängste schüren. Überhaupt stünde ein Wechsel im Diskursmodus einer Demokratie gut zu Gesicht: weg von emotionalen Appellen und starken Behauptungen hin zu rationalen Argumenten und sorgfältigen Begründungen. Auf diese Weise dürfte manches Problem seinen attestierten sicherheitspolitischen Status einbüßen.

46 Vgl.: Kohnert, Dirk: Afrikanische Migranten vor der „Festung Europa“ (GIGA Focus 12/2006), S. 4-6. 